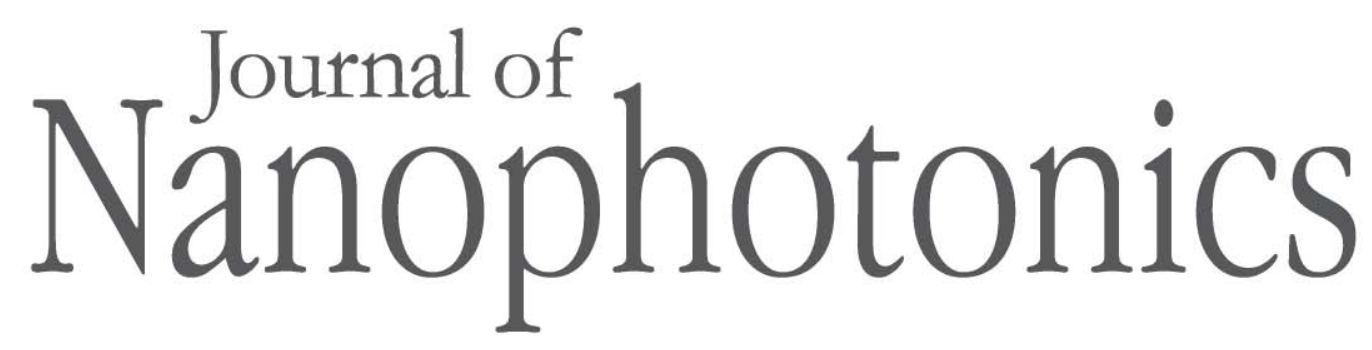

SPIEDigitalLibrary.org/jnp

\title{
Enhanced transmission of electromagnetic waves through split-ring resonator-shaped apertures
}

Levent Sahin

Koray Aydin

Gonul Turhan Sayan Ekmel Ozbay 


\title{
Enhanced transmission of electromagnetic waves through split-ring resonator-shaped apertures
}

\author{
Levent Sahin, ${ }^{\text {a,d }}$ Koray Aydin, ,a,c Gonul Turhan Sayan, \\ and Ekmel Ozbay ${ }^{\text {a,d }}$ \\ a Bilkent University, Nanotechnology Research Center, Bilkent, 06800, Ankara, Turkey \\ lsahin@bilkent.edu.tr \\ ${ }^{\mathrm{b}}$ Middle East Technical University, Department of Electrical and Electronics Engineering, \\ 06531, Ankara, Turkey \\ ${ }^{\mathrm{c}}$ California Institute of Technology, Thomas J. Watson Laboratory of Applied Physics, \\ Pasadena, California 91125 \\ ${ }^{\mathrm{d}}$ Bilkent University, Department of Electrical and Electronics Engineering, \\ 06800, Ankara, Turkey
}

\begin{abstract}
The design of aperture shape is a promising approach for enhanced transmission through a subwavelength aperture. We designed split-ring-resonator (SRR)-shaped apertures in order to increase the transmission through subwavelength apertures by making use of the strong localization of the electromagnetic field in SRR-shaped apertures. We obtained a promising result of 104-fold enhancement by utilizing SRR-shaped apertures. It is possible to use these proposed structures at optical frequencies by making several modifications such as decreasing the sharpness of edges and increasing the gap width. Since SRRs are already being realized at optical frequencies, our proposed SRR-shaped aperture structures are promising candidates for novel applications. @ 2011 Society of Photo-Optical Instrumentation Engineers (SPIE). [DOI: 10.1117/1.3599873]
\end{abstract}

Keywords: metamaterials; sub-diffraction limit; nanophotonics; apertures; wave propagation; transmission and absorption.

Paper 11010SSRR received Jan. 21, 2011; revised manuscript received May 23, 2011; accepted for publication May 24, 2011; published online Jun. 21, 2011.

\section{Introduction}

The study and debates on the characterization of wave propagation through a hole in a screen have been ongoing for centuries. In the middle of the 17th century, Grimaldi described diffraction from a circular aperture, which contributed to the foundation of classical optics. ${ }^{1}$ As classical optics suggests, Grimaldi's description was only for a circular aperture much larger than a wavelength. In 1944, Bethe gave a theoretical characterization of electromagnetic wave transmission through a subwavelength circular hole (aperture) in a perfect conductor metal screen of zero thickness, and suggested that the amplitude of the transmitted wave is proportional to the square of the area of a subwavelength aperture with $k \cdot(r / \lambda)^{4}$, where the radius $(r)$ of the aperture is much smaller than the wavelength $(\lambda)$ of the incident electromagnetic wave $(r \ll \lambda){ }^{2}$ In other words, the transmitted wave is proportional to the square of the aperture area and is inversely proportional to the fourth power of the excitation signal's wavelength. According to Bethe's ideal theoretical structure, the transmission spectra for an aperture of radius $r \ll \lambda$ has a rapidly decreasing transmission with the increased wavelength of an incident field by a power of 4 . Bethe's theory holds true for idealized situations, in which the enhancement of electromagnetic wave propagation beyond the limit of Bethe's suggestion could be obtained by utilizing novel methods to improve the coupling of an incident wave and aperture. ${ }^{3-6}$

$1934-2608 / 2011 / \$ 25.00$ @ 2011 SPIE 
The advances in characterization and fabrication techniques enabled researchers to work more on decreasing the severe effects of extremely high transmission loss and the diffraction of light through a subwavelength aperture. In 1998, "extraordinary optical transmission (EOT)" phenomena were reported by the seminal work of Ebbesen et al. ${ }^{7}$ It was shown that the coupling of light with the surface plasmons (SPs) of a two-dimensional array of sub-wavelength holes in turn yields a strong enhancement of transmitted light, which is above the limit of Bethe's prediction. This new era has attracted the interest of researchers on the enhancement of "light/electromagnetic wave" transmission through sub-wavelength apertures. The following works ${ }^{8-11}$ on the characterization of EOT phenomenon approved the involvement of surface plasmons, and also showed that the interplay between light and the resonant excitation of SPs, which are induced by a periodic array of holes, causes the EOT. The EOT phenomenon and new fabrication techniques opened up significant opportunities to new research areas and applications, such as new probes for the scanning near-field optical microscope (SNOM) ${ }^{12}$ near-field optical recording, ${ }^{13}$ nanoscale lithography, ${ }^{14-16}$ an ultrafast miniature photodetector ${ }^{17}$ and novel improved microstrip and compact waveguide filters. ${ }^{18-21}$

Surface plasmons are the resonant excitations that are caused by the coupling between the free surface charges of metal and the incident electromagnetic field at the interface, separating a dielectric medium with a metal. Since metals at microwave frequencies possess very high conductance [metals behave similar to perfect electric conductors (PECs) in the microwave spectrum]; surface plasmons are not supposed to exist at the microwave frequency regime. Therefore, there has been a debate going on regarding a new way of explanation for EOT at microwave frequencies. ${ }^{22,23}$ In this approach, SPs are not considered to exist in the microwave regime, in which "spoof surface plasmons" that are very similar to SPs are formed between the dielectric substrate and metal at microwave frequencies. ${ }^{24}$ SPs and spoof SPs serve in a similar way for guiding the electromagnetic wave to the aperture in order to, in turn, obtain an enhanced transmission. In our present work, we obtained enhanced transmission at microwave frequencies, wherein the metals act as perfect conductors. Therefore, localized surface plasmons do not contribute to the enhancement process. In addition, since grating structures are not used in our approach, there is no significant contribution of surface waves to enhanced transmission. As we will show in the following, the strong localized fields around split-ring resonator (SRR)based novel structures couple to the incident electromagnetic field through a resonant process, causing a strong extraordinary transmission of electromagnetic waves.

\section{Design and Fabrication of Sub-Wavelength Aperture Structures}

We investigated novel structures that are utilized for the enhanced transmission of electromagnetic waves through a single subwavelength aperture. Previous studies have shown that an aperture with rectangular ${ }^{24}$ and elliptical ${ }^{25}$ shapes in turn yield enhanced transmission for the specific polarization of an incident electromagnetic wave. Furthermore, enhanced transmission through a subwavelength aperture, by utilizing SRR structures, was previously shown theoretically ${ }^{26}$ and experimentally. ${ }^{27}$

The manipulation of the aperture shapes is a promising approach for enhanced transmission through subwavelength apertures. In the present work, we utilized SRR-shaped apertures in order to increase the transmission through a subwavelength aperture by using the strong localization characteristics of SRR structures. ${ }^{28}$

The idea of an SRR-shaped aperture stems from Babinet's principal. ${ }^{29,30}$ By using Babinet's principle, we can anticipate that the reflected fields of the SRR structure for a given incident polarization are supposed to be similar to the transmittance spectra of the complementary SRRshaped aperture for orthogonal polarization. Since SRR is a strong resonator structure, there exists a dip in the transmittance spectra at the resonance frequency. The dip in the transmission spectrum of the SRR corresponds to a peak in the reflection spectrum. Therefore, we can utilize this peak in order to obtain enhanced transmission through subwavelength apertures according to Babinet's principle. 


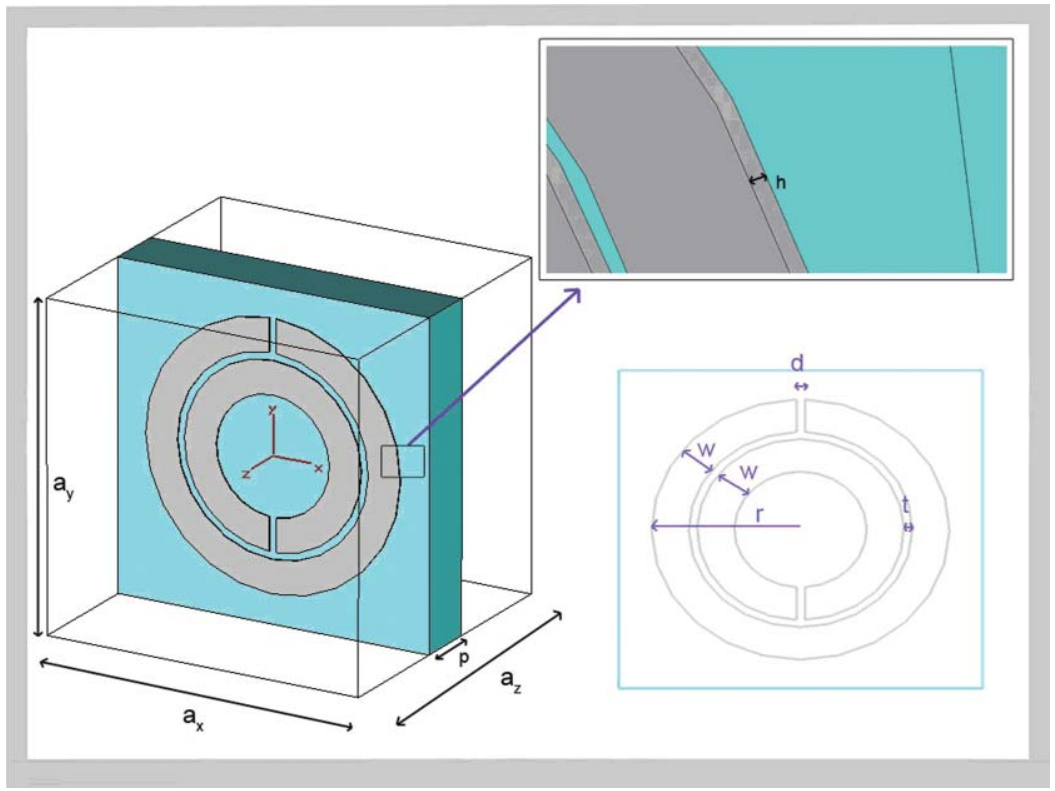

Fig. 1 Unit cell of the SRR structure.

First, we performed simulations on an SRR structure. Split-ring resonator structures comprise two concentric copper rings with splits oriented at opposite sides. Figure 1 shows a schematic representation of the SRR that we used. The gap between the inner and outer rings $(t)$, and the width of the splits (d), are $0.2 \mathrm{~mm}$, the metal width (w) is $0.9 \mathrm{~mm}$, and the outer radius of the SRR structure is $3.6 \mathrm{~mm}$. SRR is deposited on a commercial FR4 dielectric board with a thickness of $1.6 \mathrm{~mm} .{ }^{31,32}$

The simulation of the single SRR structure was carried out by modeling a waveguidelike structure. Namely, two waveguide ports were used to obtain S-21 data. Open boundary conditions were employed along the propagation direction $(+x$ and $-x)$. Electric $\left(E_{t}=0\right)$ boundary conditions were used for the $-y$ and $+y$ direction. In addition, magnetic $\left(H_{t}=0\right)$ boundary conditions were used for the $-z$ and $+z$ direction.

Figure 2(b) shows the calculated transmission and reflection spectrum of the SRR structure. There is a peak in the reflection spectrum. Therefore, we expected to have a peak in the transmission spectrum of the SRR-shaped aperture for the orthogonal polarization of an incident wave (in accordance with Babinet's principle).

The numerical simulations were performed by the commercially available software, CST Microwave Studio. We modeled the complete scene in a real electromagnetic measurement setup in order to decrease the deviations from the experimental results. Namely, two waveguide antennas, including the waveguide feed structures and the device under test (DUT), were modeled (Fig. 3). Open boundary conditions were applied along all directions and PEC approximation was used for metal parts in the simulations. Since metals are very good conductors at the microwave spectrum and the open boundary conditions can be obtained by using absorbers around the measurement setup, the approximations that are used in the simulations are reasonable and acceptable.

The metallic plate with an aperture is placed $0.1 \mathrm{~mm}$ away from the transmitter antenna and the receiver antenna is located $5 \mathrm{~cm}$ away from the transmitter antenna. The operating wavelengths of the structures are between 5 and $10 \mathrm{~cm}$. Since subwavelength resonances can be obtained when the input is trigerred at close proximity of aperture, we put the transmitting waveguide at a subwavelength distance and we optimized the distance by making the resonance peak maximum. On the other hand, the sample and receiving antenna should not be very close to avoid unwanted coupling at the output signal. Therefore, we put the receiving antenna 


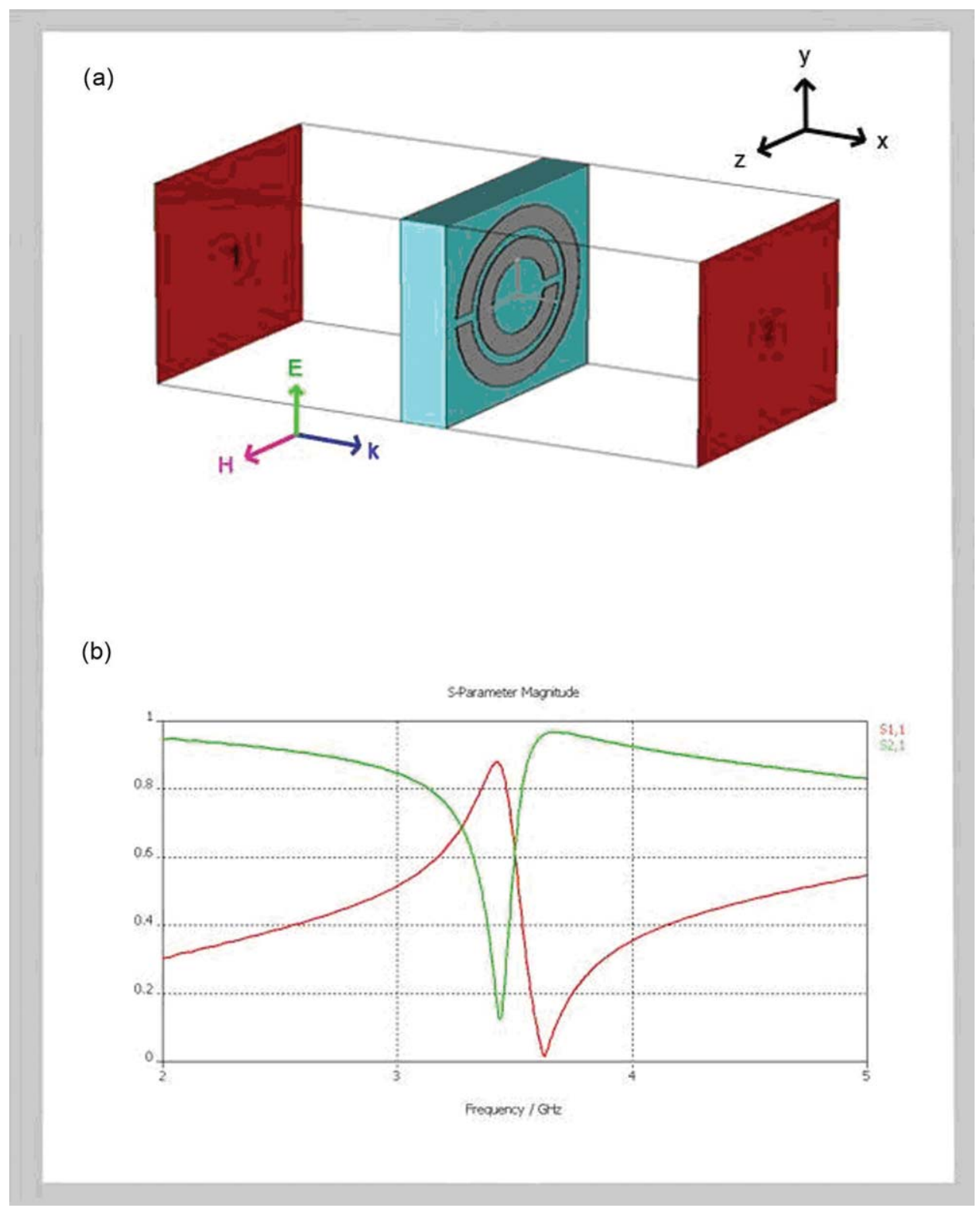

Fig. 2 (a) Schematic representation of the SRR_A structure as given in Sec. 3. (b) Transmission (S21) and reflection (S11) spectra of the SRR_A structure.

approximately at a wavelength distance. Also, we cannot put the receiving antenna at a further distance, because our measurement devices will not be able to detect very small signals.

The SRR-shaped aperture is complementary to an exact replica of the SRR_A structure. The SRR-shaped aperture is modeled by subtracting the SRR_A structure from a square metal plate with a size of $L \times L(L=200 \mathrm{~mm})$. The metal plate is composed of a copper metal with a thickness of $30 \mu \mathrm{m}$ and a 1.6-mm thick commercial FR-4 PCB substrate. Figure 4 shows a schematic representation of the SRR-shaped aperture that we used. The gap between the inner and outer rings $(\mathrm{t})$; and the width of the splits (d) are $0.2 \mathrm{~mm}$, the ring width (w) is $0.9 \mathrm{~mm}$, and the outer radius of the SRR structure is $3.6 \mathrm{~mm}$ (SRR_A type configuration). The dielectric constant of the FR-4 dielectric substrate is taken as $\varepsilon=3.6$ with a tangent loss of $\delta=0.01$. 
Sahin et al.: Enhanced transmission of electromagnetic waves through split-ring resonator-shaped apertures

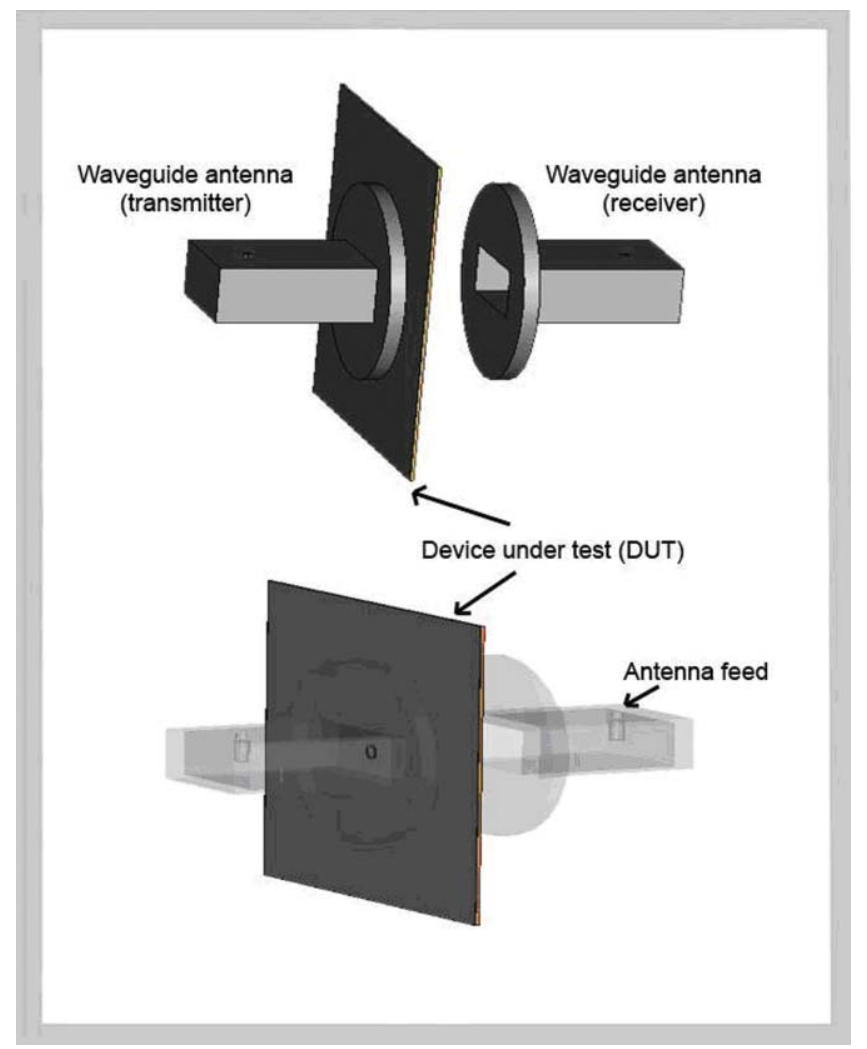

Fig. 3 Schematics of two waveguide antennas, including the waveguide feed structures and the DUT.

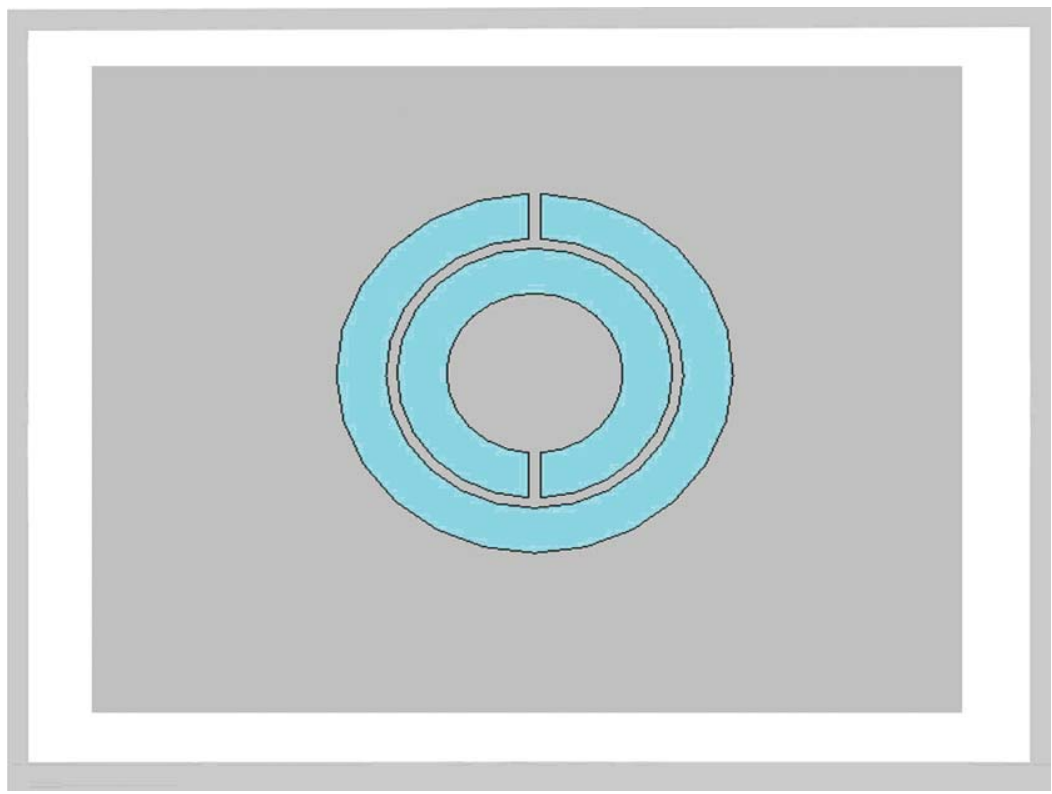

Fig. 4 Schematic representation of the SRR-shaped aperture (dark-colored parts are the metal plate and the light-colored parts are the gaps). 


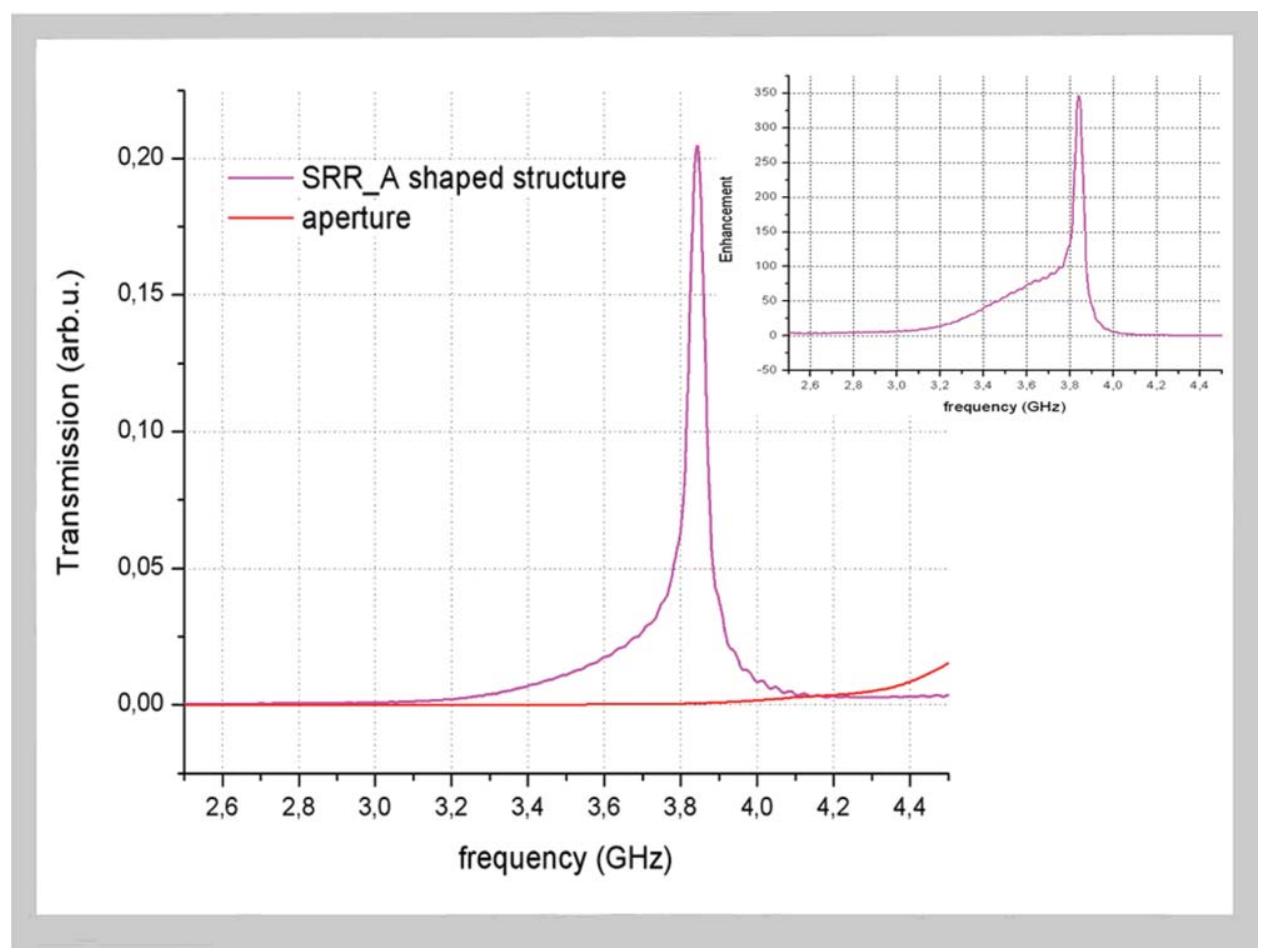

Fig. 5 The calculated transmission spectrum of the SRR-shaped structure and a single aperture. The enhancement spectrum obtained by employing the SRR-shaped structure is given in the inset.

Figure 5 shows the calculated transmission result of the SRR-shaped aperture, which demonstrated an extraordinary transmission peak.

The extraordinary transmission result that was observed at the response of SRR-shaped aperture confirms that our expectation, which is based on Babinet's principle, holds. This novel approach opens up significant opportunities to utilize several resonator structures for obtaining extraordinary transmission through subwavelength apertures. In the present work, we investigated SRR-shaped apertures for increasing the transmission through a subwavelength aperture.

The simulation results of the SRR-shaped structure and a single aperture show significant enhancement in the transmission of a single subwavelength aperture (Fig. 5).

The enhancement in the transmission through an aperture is defined as the ratio of the field intensity of the transmitted electromagnetic wave through an SRR-shaped aperture to that through the aperture only. The inset of Fig. 5 shows the enhancement that was obtained by manipulating the shape of the aperture as the SRR-shaped aperture. We obtained a 346-fold transmission enhancement at $3.84 \mathrm{GHz}$. It is noteworthy that the enhancement peak is very close to the resonance frequency of the SRR structure. ${ }^{27}$ The resonance coupling of the incident electromagnetic wave to the SRR-shaped aperture causes the strong localization of the electric field at the splits and gaps of the SRR-shaped aperture. Therefore, the resonator characteristic of SRR is responsible for the confined electromagnetic waves in the SRR-shaped aperture. We checked the role of the SRR characteristics on the enhanced transmission through an SRRshaped aperture by closing the split regions of the structure, in which the structure became a closed ring resonator (CRR)-shaped aperture [Fig. 6(a)]. Since the resonant behavior of SRR diminishes by closing the splits, the role of the resonant structure of SRR on the enhancement mechanism of an SRR-shaped aperture is evident [Fig. 6(b)]. 


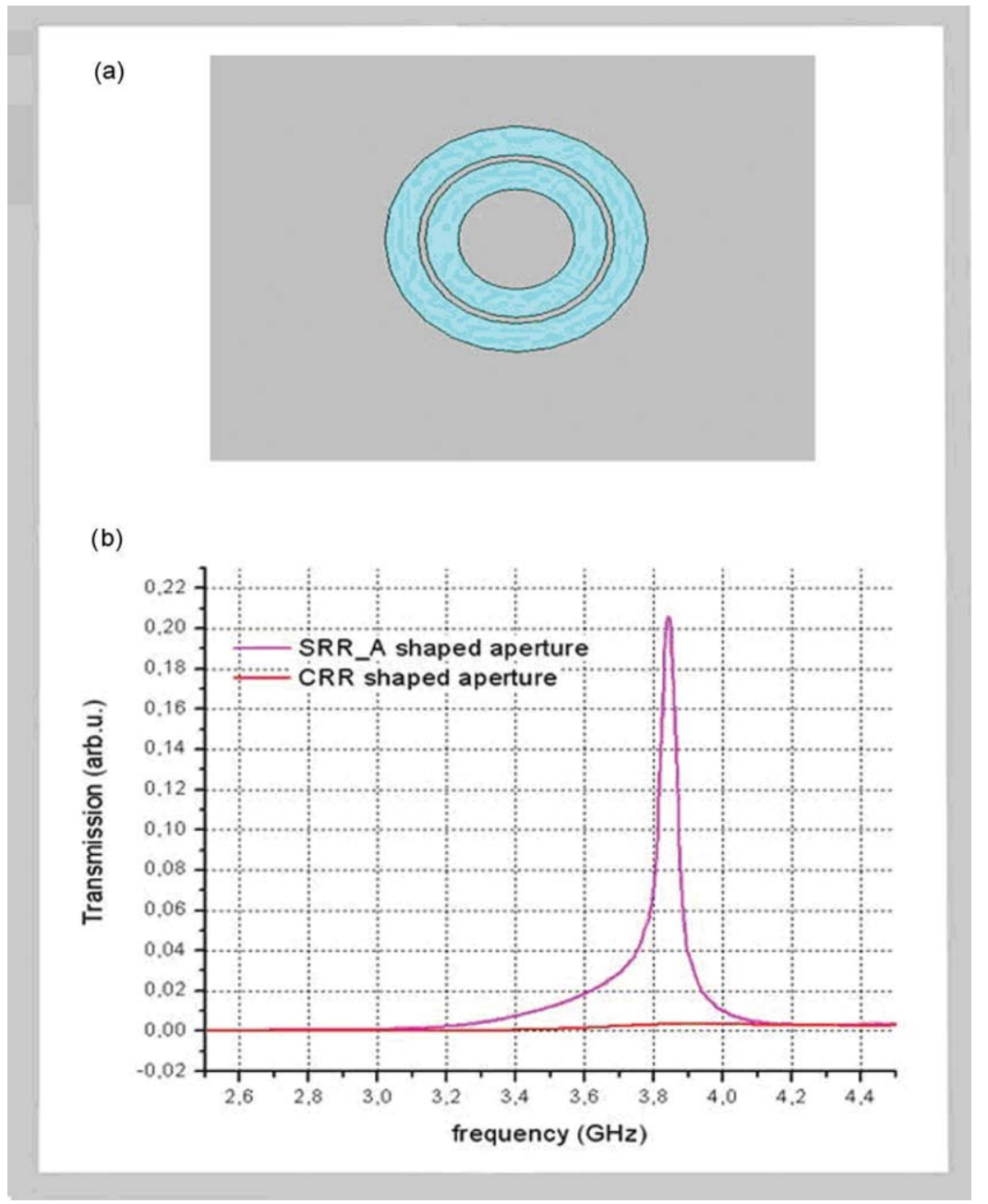

Fig. 6 (a) Schematic representation of the CRR-shaped aperture. (b) Transmission spectrum of the CRR-shaped aperture and the SRR-shaped aperture.

After the successful demonstration of enhanced transmission through SRR-shaped aperture, we simulated, manufactured, and measured several other types of split ring resonator structures in order to better characterize the novel approach/structure that we proposed. We performed a parametric study of an SRR-shaped aperture, and we also investigated single-ring SRR-shaped apertures. We mostly used square-shaped structures due to ease of fabrication for the applications at optical frequencies. The schematic representations of the proposed structures are shown in Fig. 7.

We investigated the enhanced microwave transmission through subwavelength apertures for 12 different samples. The maximum length of all the samples was $7.2 \mathrm{~mm}$, which corresponds to approximately $0.1 \lambda$ at the resonance frequency of the SRR structures ( $\sim 3$ to $4 \mathrm{GHz}$ ).

Figure $8(\mathrm{a})$ is the numerical simulation results of the transmission intensity of the electromagnetic wave that propagates through the proposed SRR-shaped apertures.

As can be seen in Fig 8, the transmission through the subwavelength aperture is significantly increased when SRR-shaped apertures are placed instead of only apertures. Moreover, we observed that the annular apertures (samples 3,8 , and 12) transmit electromagnetic waves very close to the single aperture sample (sample 4), which indicates that SRR-shaped apertures show superior performance versus annular apertures in terms of subwavelength aperture transmission. 


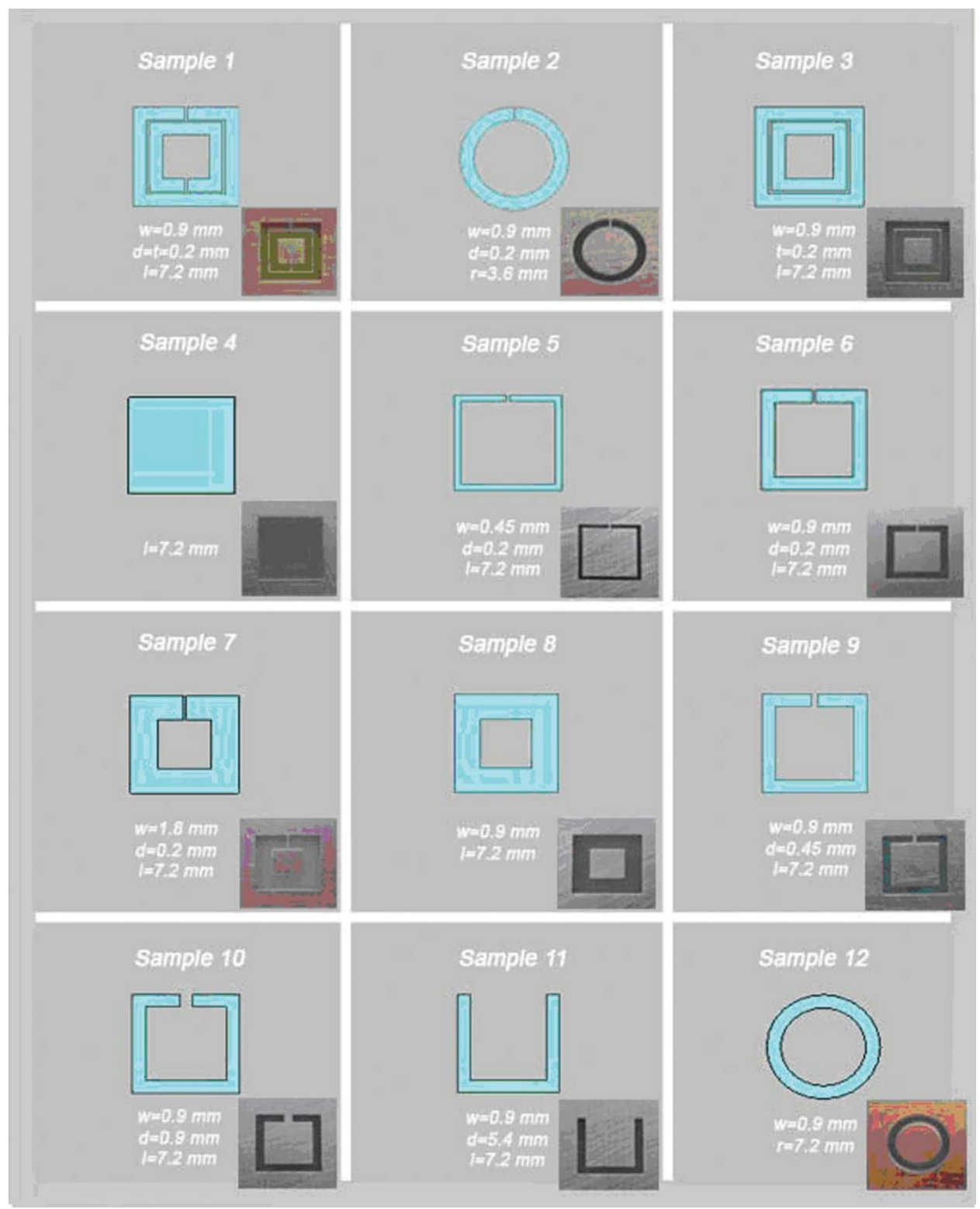

Fig. 7 The schematics of the proposed SRR-shaped aperture structures (gray parts are the metal plate and the blue parts are the gaps). The insets demonstrate the fabricated samples.

This also shows the effect of the resonant coupling mechanism via SRR-shaped apertures. The resonance of SRR enables the coupling of the incident wave to the transmitted wave. Figure 8(b) shows the enhancement values of the simulated structures.

Moreover, some of the peaks that are shown in Fig. 8(a) are not available in Fig. 8(b), because transmission through a subwavelength aperture (sample 4 and circular aperture) increases with the power of 4 as the frequency increases. Therefore, certain peaks at the end of the interested frequency spectrum disappear in the enhancement plot as a result of an increase in the transmission through the aperture at higher frequencies.

After demonstrating this significant enhancement by the proposed structures, we fabricated and experimentally measured the samples in order to confirm the performance of novel structures. 


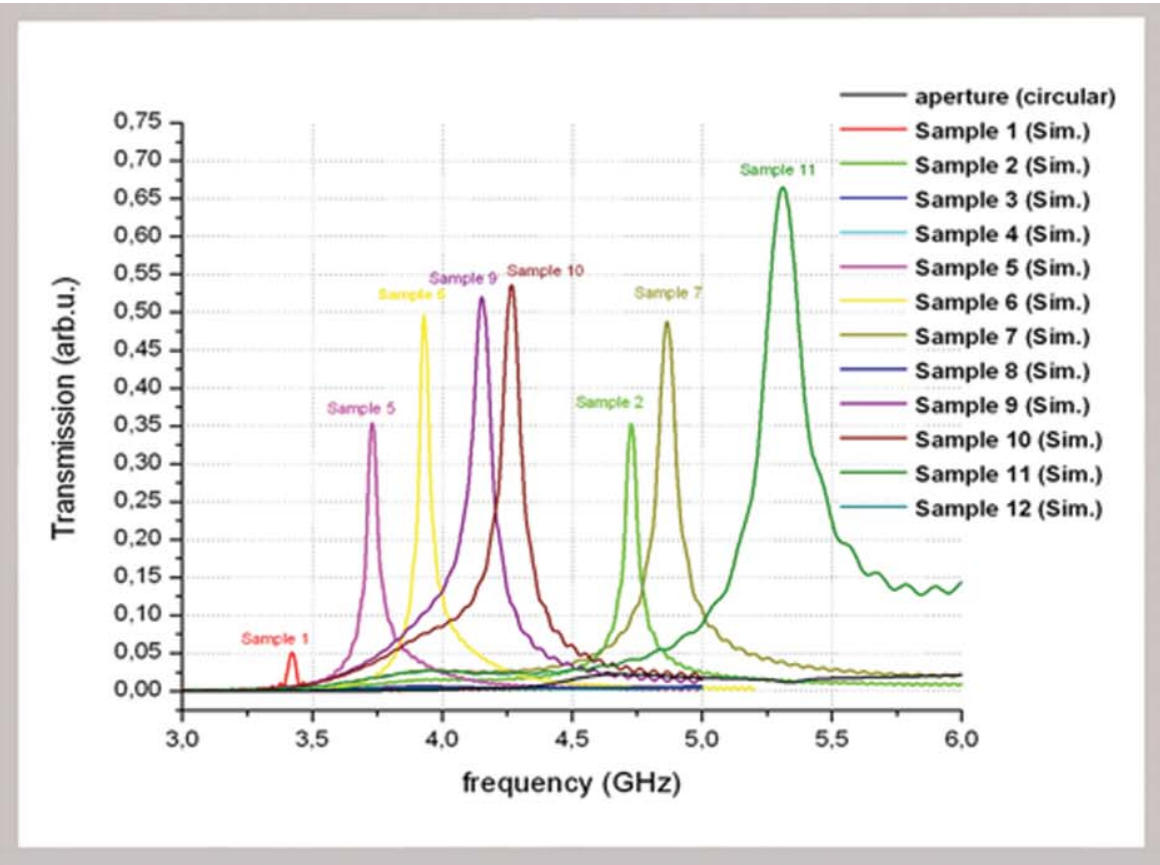

a)
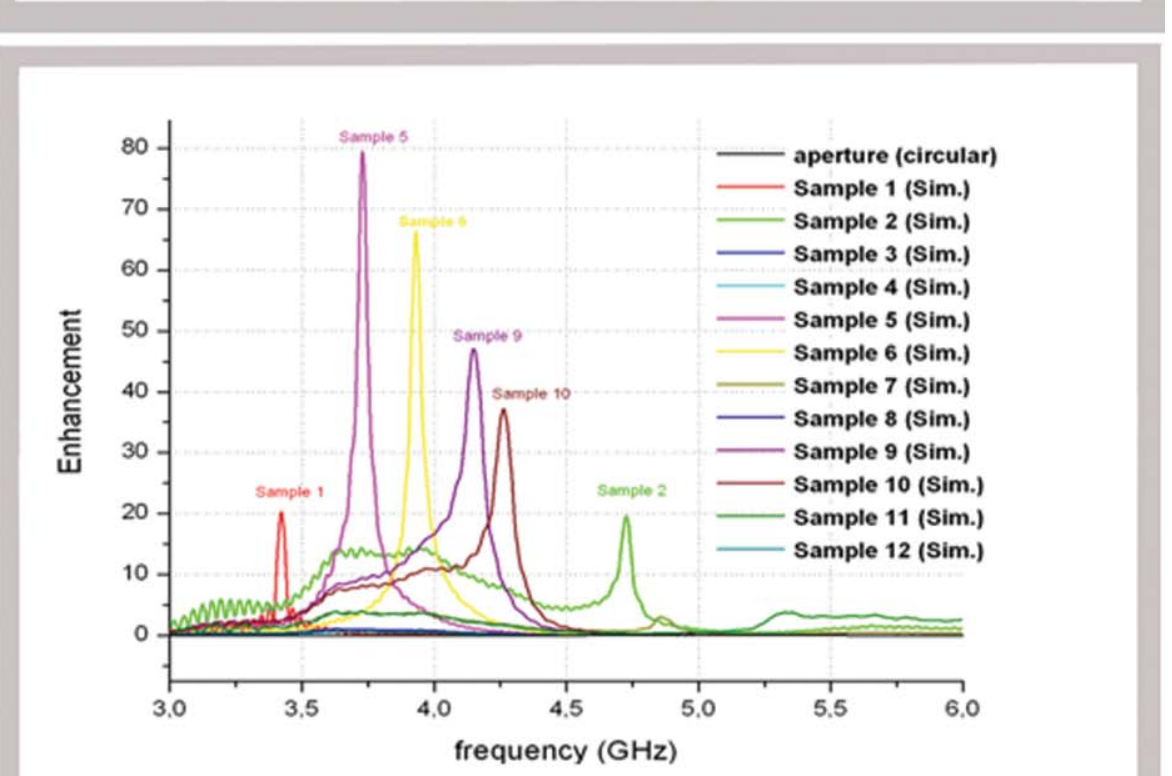

b)

Fig. 8 Calculated (a) transmission results and (b) enhancement results of the proposed SRRshaped apertures that are shown in Fig. 7.

We used an experimental setup comprising an Agilent N5230A portable network analyzer, two waveguide ports, and the proper SMA cables. The waveguide ports were used as the transmitter and the receiver and were connected to the network analyzer with SMA cables. In the measurements, the metallic plate with an aperture was placed $0.1 \mathrm{~mm}$ away from the transmitter antenna and the receiver antenna was located $5 \mathrm{~cm}$ away from the transmitter antenna, just as we modeled in the numerical simulations. Waveguide ports were employed as transmitter and receiver antennas. The propagation of the incident polarized EM wave was along the $z$-axis, whereas the E-field was along the $y$ direction, and the $\mathrm{H}$-field was along the $x$ direction. 


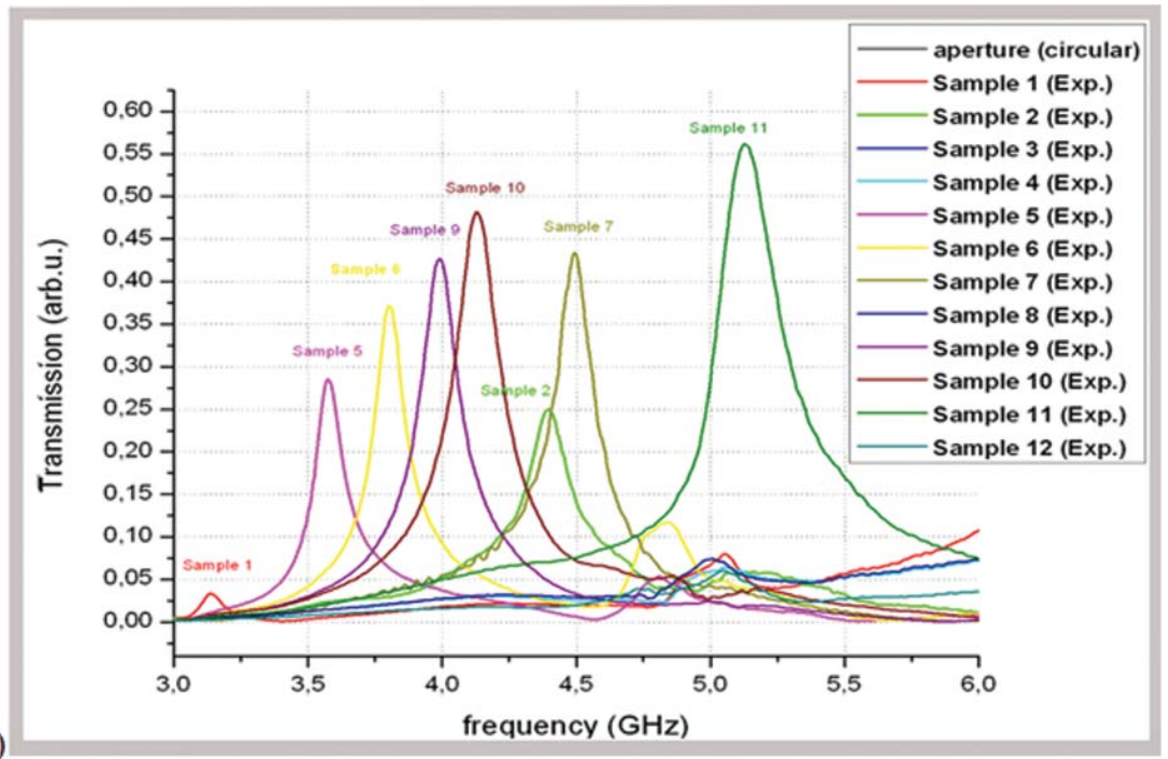

(a)

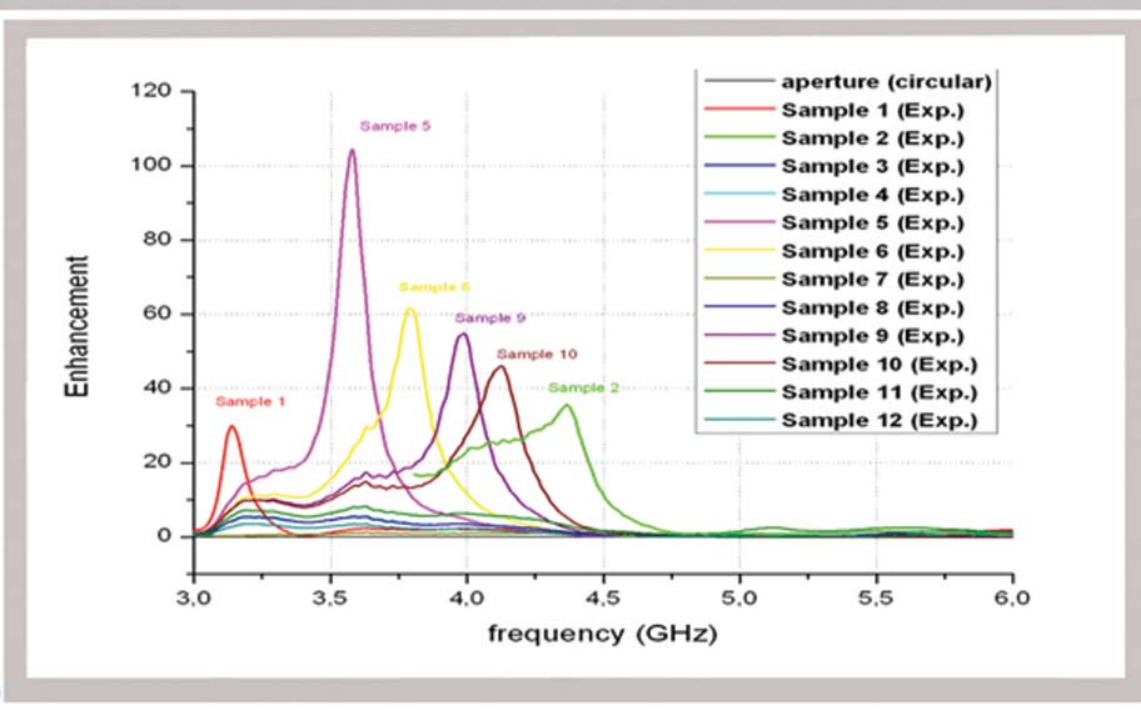

Fig. 9 Measured (a) transmission spectrum and (b) enhancement spectrum of the proposed SRR-shaped apertures.

The insets of Fig. 7 represent the fabricated structures. These samples were fabricated by using a computer numerical control (CNC) machine with a process resolution of $0.1 \mathrm{~mm}$. Figure 9(a) displays the measured transmission spectra of the 12 samples. The enhancement values of the experimentally measured samples are shown in Fig. 9(b).

The experimental and numerical transmission results are in good agreement, which indicates that our proposed structures for achieving significant enhancement are properly designed for simulations. Table 1 is a summary of transmission and enhancement values of the proposed structures.

Sample-4 is the reference sample for our measurements. As we stated above, the subwavelength apertures transmit very poorly and diffract electromagnetic radiation in all directions. Therefore, all of the samples were designed on a $20 \mathrm{~cm} \times 20 \mathrm{~cm}$ metal plate in order to better collect the transmitted wave and better isolate the incident wave and transmitted wave.

Sample-1 has the highest transmission wavelength (lowest radius to the wavelength ratio) as a result of the additional splits and rings compared to the other samples. Furthermore, we 
Table 1 Measured transmission and enhancement values of proposed structures.

\begin{tabular}{|c|c|c|c|c|c|c|c|c|c|}
\hline & \multirow{2}{*}{$\begin{array}{c}\mathbf{w} \\
(\mathrm{mm})\end{array}$} & \multirow{2}{*}{$\begin{array}{c}\mathrm{d} / \mathrm{t} \\
(\mathrm{mm})\end{array}$} & \multirow{2}{*}{$\begin{array}{c}\mathbf{r} / \mathbf{l} \\
(\mathrm{mm})\end{array}$} & \multicolumn{2}{|c|}{$\begin{array}{c}\text { peak frequency } \\
\text { (GHz) }\end{array}$} & \multicolumn{2}{|c|}{$\begin{array}{c}\text { Transmission } \\
\text { peak (arb. units) }\end{array}$} & \multicolumn{2}{|c|}{$\begin{array}{c}\text { Enhancement } \\
\text { peak (arb. units) }\end{array}$} \\
\hline & & & & Sim. & Exp. & Sim. & Exp. & Sim. & Exp. \\
\hline Sample 1 & 0.9 & $0.2 / 0.2$ & 7.2 & 3.42 & 3.13 & 0.051 & 0.033 & 20 & 30 \\
\hline Sample 2 & 0.9 & 0.2 & 3.6 & 4.73 & 4.41 & 0.35 & 0.25 & 20 & 36 \\
\hline Sample 3 & 0.9 & $-/ 0.2$ & 7.2 & $\mathrm{~N} / \mathrm{A}$ & $\mathrm{N} / \mathrm{A}$ & $\mathrm{N} / \mathrm{A}$ & $\mathrm{N} / \mathrm{A}$ & $\mathrm{N} / \mathrm{A}$ & $\mathrm{N} / \mathrm{A}$ \\
\hline Sample 4 & - & - & 7.2 & $\mathrm{~N} / \mathrm{A}$ & $\mathrm{N} / \mathrm{A}$ & $\mathrm{N} / \mathrm{A}$ & $\mathrm{N} / \mathrm{A}$ & $\mathrm{N} / \mathrm{A}$ & $\mathrm{N} / \mathrm{A}$ \\
\hline Sample 5 & 0.45 & 0.2 & 7.2 & 3.73 & 3.58 & 0.35 & 0.29 & 80 & 104 \\
\hline Sample 6 & 0.9 & 0.2 & 7.2 & 3.93 & 3.79 & 0.49 & 0.37 & 66 & 62 \\
\hline Sample 7 & 1.8 & 0.2 & 7.2 & 4.87 & 4.49 & 0.49 & 0.43 & $\mathrm{~N} / \mathrm{A}$ & $\mathrm{N} / \mathrm{A}$ \\
\hline Sample 8 & 0.9 & - & 7.2 & N/A & N/A & $\mathrm{N} / \mathrm{A}$ & $\mathrm{N} / \mathrm{A}$ & $\mathrm{N} / \mathrm{A}$ & $\mathrm{N} / \mathrm{A}$ \\
\hline Sample 9 & 0.9 & 0.45 & 7.2 & 4.15 & 3.99 & 0.52 & 0.43 & 47 & 55 \\
\hline Sample 10 & 0.9 & 0.9 & 7.2 & 4.26 & 4.13 & 0.54 & 0.48 & 37 & 46 \\
\hline Sample 11 & 0.9 & 5.4 & 7.2 & 5.31 & 5.13 & 0.66 & 0.56 & $\mathrm{~N} / \mathrm{A}$ & $\mathrm{N} / \mathrm{A}$ \\
\hline Sample 12 & 0.9 & - & 7.2 & $\mathrm{~N} / \mathrm{A}$ & $\mathrm{N} / \mathrm{A}$ & $\mathrm{N} / \mathrm{A}$ & $\mathrm{N} / \mathrm{A}$ & $\mathrm{N} / \mathrm{A}$ & $\mathrm{N} / \mathrm{A}$ \\
\hline
\end{tabular}

observed that as the ring width decreases, the transmission enhancement and transmission peak frequency increases; this is because the decreased width of the SRR-shaped apertures increases the coupling between the plate at the center and the surrounding large plane, which increases the resonant coupling.

We also investigated the effect of a split width on the enhancement peak. The enhancement value of the samples decreases as we increase the split width. Furthermore, the resonance peak frequency increases with the increasing split width. The split width is very crucial for the resonance of SRR. The effect of increasing the split width is also very important for the application of our proposed structures at optical frequencies. Since the fabrication of sample-11 is the easiest among all the others, it will be a better choice for higher frequencies compared to structures with complicated shapes. As we can see from the results, we obtained a significant transmission peak, which is very promising for higher frequency applications.

Finally, we investigated the effect of the shape of SRR on the enhancement. We fabricated sample- 2 and sample- 12 for observing the effect of the SRR-type. The transmission of sample-12 is as we expected, very low. However, we obtained a significant enhancement (35.58fold) by only utilizing a small wire that makes the structure an SRR-shaped structure (sample-2). We used a circular aperture (radius $3.6 \mathrm{~mm}$ ) as the reference sample for circular SRR-shaped aperture structures for a fair comparison.

The metal that we used in our structures is very close to a perfect conductor at microwave frequencies. Therefore, localized surface plasmons do not contribute to the enhanced transmission. The enhancement stems from the strongly localized fields that are caused by the resonant process of SRR-shaped aperture to the subwavelength aperture.

\section{Conclusion}

In summary, we demonstrated several novel structures to increase the transmission through a subwavelength aperture. We obtained a 104-fold enhancement at microwave frequencies by utilizing SRR-shaped apertures. Enhanced transmission through a subwavelength aperture has become a current subject of intense research due to a wide range of applications of this phenomenon. ${ }^{33-35}$ As SRRs are already being realized at optical frequencies, ${ }^{31,32}$ it is possible to use these SRR-based structures for new optical applications, such as new probes for near-field optical recording, SNOM, nanoscale lithography, and ultrafast miniature photodetectors. 


\section{Acknowledgments}

This work is supported by the European Union under the projects EU-PHOME, EU-N4E and EU-ECONAM, and TUBITAK under Project 107A012. One of the authors (E.O.) also acknowledges partial support from the Turkish Academy of Sciences.

\section{References}

1. F. M. Grimaldi, "Physico-mathesis de Lumine, Coloribus, et Iride," Aliisque Sequenti Pagina Indicatis 9, 1-533, Bologna (1665).

2. H. A. Bethe, "Theory of diffraction by small holes," Phys. Rev. 66, 163-182 (1944).

3. X. Shi, L. Hesselink, and R. Thornton, "Ultrahigh light transmission through a C-shaped nanoaperture," Opt. Lett. 28(15), 1320-1322 (2003).

4. X. Shi and L. Hesselink, "Design of a $\mathrm{C}$ aperture to achieve $\lambda / 10$ resolution and resonant transmission," J. Opt. Soc. Am. 21(7), 1305-1317 (2004).

5. L. Tang, D. A. B. Miller, A. K. Okyay, J. A. Matteo, Y. Yuen, K. C. Saraswat, and L. Hesselink, "C-shaped nanoaperture-enhanced germanium photodetector," Opt. Lett. 31(10), 1519-1521 (2006).

6. P. Hansen, L. Hesselink, and B. Leen, "Design of a subwavelength bent C-aperture waveguide," Opt. Lett. 32(12), 1737-1739 (2007).

7. T. W. Ebbesen, H. J. Lezec, H. F. Ghaemi, T. Thio, and P. A. Wolff, "Extraordinary optical transmission through sub-wavelength hole arrays," Nature (London) 391, 667-669 (1998).

8. T. D. Visser, "Plasmonics: Surface plasmons at work?," Nat. Phys. 2, 509-510 (2006).

9. H. F. Schouten, N. Kuzmin, G. Dubois, T. D. Visser, G. Gbur, P. F. A. Alkemade, H. Blok, G. W. Hooft, D. Lenstra, and E. R. Eliel, "Plasmon-assisted two slit two-slit transmission: Young's experiment revisited," Phys. Rev. Lett. 94, 053901 (2005).

10. K. L. Klein Koerkamp, S. Enoch, F. B. Segerink, N. F. van Hulst, and L. Kuipers, "Strong influence of hole shape on extraordinary transmission through periodic arrays of subwavelength holes," Phys. Rev. Lett. 92, 183901 (2004).

11. H. Caglayan, I. Bulu, and E. Ozbay, "Extraordinary grating-coupled microwave transmission through a subwavelength annular aperture," Opt. Express 13, 1666-1671 (2005).

12. M. J. Levene, J. Korlach, S. W. Turner, M. Foquet, H. G. Craighead, and W. W. Webb, "Zero-mode waveguides for single molecule analysis at high concentrations," Science 299, 682-686 (2003).

13. G. Gbur, H. F. Schouten, and T. D. Visser, "Achieving super resolution in near-field optical data readout systems using surface plasmons," Appl. Phys. Lett. 87, 191109 (2005).

14. W. Srituravanich, N. Fang, C. Sun, Q. Luo, and X. Zhang, "Plasmonic nanolithography," Nano Lett. 4, 1085-1088 (2004).

15. X. Luo and T. Ishihara, "Sub-100nm photolithography based on plasmon resonance," Jpn. J. Appl. Phys. 43, 4017-4021 (2004).

16. D. B. Shao and S. C. Che, "Surface-plasmon-assisted nanoscale photolithography by polarized light," Appl. Phys. Lett. 86, 253107 (2005)

17. T. Ishi, J. Fujikata, K. Makita, T. Baba, and K. Ohashi, "Si nano-photodiode with a surface plasmon antenna," Jpn. J. Appl. Phys. 44, L364-L366 (2005).

18. F. Falcone, T. Lopetegi, J. D. Baena, R. Marqués, and F. Martin, "Effective negative-eps stopband microstrip lines based on complementary split ring resonators," IEEE Microw. Wirel. Compon. Lett. 14(6), 280-282 (2004).

19. N. Ortiz, J. D. Baena, M. Beruete, F. Falcone, M. A. G. Laso, T. Lopetegi, R. Marque's, F. Martin, J. Garcia-Garcia, and M. Sorolla, "Complementary split-ring resonator for compact waveguide filter design," Microwave Opt. Technol. Lett. 46(1), 88-92 (2005).

20. M. Aznabet, M. Beruete, M. Navarro-Cía, O. El Mrabet, F. Falcone, N. Aknin, M. Essaaidi, and M. Sorolla, "Metamaterial multiresonances in waveguide and metasurfaces," Microwave Opt. Technol. Lett. 50(11), 2825-2827 (2008). 
21. R. Marqués, J. D. Baena, M. Beruete, F. Falcone, T. Lopetegi, M. Sorolla, F. Martín, and J. Garcia, "Ab initio analysis of frequency selective surfaces based on conventional and complementary split ring resonators," J. Opt. A, Pure Appl. Opt. 7(2), s38-s43 (2005).

22. J. B. Pendry, L. Martin-Moreno, and F. J. Garcia-Vidal, "Mimicking surface Plasmons with structured surfaces," Science 305, 847-848 (2004).

23. F. J. Garcia-Vidal, L. Martin-Moreno, and J. B. Pendry, "Surfaces with holes in them: New plasmonic metamaterials," J. Opt. Pure Appl. Opt. 7, S97-S101 (2005).

24. F. J. Garcia-Vidal, E. Moreno, J. A. Porto, and L. Martin-Moreno, "Transmission of light through a single rectangular hole," Phys. Rev. Lett. 95, 103901 (2005).

25. R. Gordon and A. Brolo, "Increased cut-off wavelength for a sub-wavelength hole in a real metal," Opt. Express 13, 1933-1938 (2005).

26. R. Marqués, F. Mesa, J. Martel, and F. Medina, "Comparative analysis of edge- and broadside-coupled Split ring resonators for metamaterial design-theory and experiments," IEEE Trans. Antennas Propag. 51(10), 2572-2581 (2003).

27. K. Aydin, A. O. Cakmak, L. Sahin, Z. Li, F. Bilotti, L. Vegni, and E. Ozbay, "Split-ringresonator-coupled enhanced transmission through a single subwavelength aperture," Phys. Rev. Lett. 102, 013904 (2009).

28. K. Aydin, K. Guven, M. Kafesaki, C. M. Soukoulis, and E. Ozbay, "Investigation of magnetic resonances for different split-ring resonator parameters and designs," New $J$. Phys. 7, 168-184 (2005).

29. R. F. Harrington, Time-Harmonic Electromagnetic Fields, pp. 365-367, IEEE Press, Wiley Interscience (2001).

30. F. Falcone, T. Lopetegi, M. A. G. Laso, J. D. Baena, J. Bonache, M. Beruete, R. Marqués, F. Martín, and M. Sorolla, "Babinet principle applied to the design of metasurfaces and metamaterials," Phys. Rev. Lett. 93, 197401 (2004).

31. T. J. Yen, W. J. Padilla, N. Fang, D. C. Vier, D. R. Smith, J. B. Pendry, D. N. Basov, and X. Zhang, "Terahertz magnetic response from artificial materials," Science 303, 1494 (2004).

32. C. Enkrich, M. Wegener, S. Linden, S. Burger, L. Zschiedrich, F. Schmidt, J. F. Zhou, T. Koschny, and C. M. Soukoulis, "Magnetic metamaterials at telecommunication and visible frequencies," Phys. Rev. Lett. 95, 203901 (2005).

33. J. Dintiger, S. Klein, and T. W. Ebbessen, "Molecule-surface plasmon interactions in hole arrays: Enhanced absorption, refractive index changes, and all optical switching," $A d v$. Mater. (Wienheim, Ger.) 18, 1267-1270 (2006).

34. I. I. Smolyaninov, A. V. Zayats, A. Stanishevsky, and C. C. Davis, "Optical control of photon tunneling through an array of nanometer-scale cylindrical channels," Phys. Rev. B 66, 205414 (2002).

35. C. Janke, J. Gómez Rivas, P. Haring Bolivar, and H. Kurz, "All-optical switching of the transmission of electromagnetic radiation through subwavelength apertures," Opt. Lett. 30, 2357-2359 (2005).

Biographies and photographs of the authors not available. 